\title{
The Multi-Agent Navigation Transformation: Tuning-Free Multi-Robot Navigation
}

\author{
Savvas G. Loizou \\ Mechanical Engineering and Materials Science and Engineering \\ Cyprus University of Technology \\ Limassol 3041, CYPRUS \\ Email: savvas.loizou@cut.ac.cy
}

\begin{abstract}
This paper proposes a novel methodology for decentralized multi-robot navigation with multiple arbitrarily shaped obstacles in 2-dimensional environments. The proposed methodology is based on the novel concepts of the Navigation Transformation and the Harmonic Function based Navigation Functions. A version of the Navigation Transformation - the Multi-Agent Navigation Transformation - is proposed in this paper to map geometrically complex topologies resulting from moving workspace entities to simple topologies enabling the construction of Harmonic Function based Navigation Functions. The resulting vector field is guaranteed to be free of local minima by construction. A construction of a candidate Multi-Agent Navigation Transformation is proposed. In addition to the theoretical guarantees, the effectiveness of the proposed methodology is demonstrated through non-trivial computer simulations utilizing the proposed construction.
\end{abstract}

\section{INTRODUCTION}

Multi-robot navigation is a problem that has been considered by the robotics community since the early 90's [17], while still being an active research topic [18, 15, 3, 8, 10].

One of the arguably most successful frameworks for robotic navigation is the Navigation Functions (NF) framework [9]. NFs provide the capability for closed-form fast-feedback based control for navigating a robot from any initial configuration to its destination configuration, while being amenable to rigorous analysis regarding convergence and stability properties. Due to the closed-form solution, the NF framework enable applications on systems with limited computational capabilities. The NF framework has been successfully extended to handle multiple robots in centralized [15] and decentralized setups [3, 16], with non-holonomic constraint [14] and has also been augmented with spacio-temporal scheduling and planning capabilities [6, 4].

One of the major points of criticism for the NF based techniques has been the issue of tuning ${ }^{1}$ Recent results on Navigation Functions based on Harmonic Potentials [12] that utilize the Navigation Transformation concept [13], provide vector fields that are free of local minima by construction, whereas automatic tuning controllers can enforce the Morse property (non-degeneracy of critical points), yielding a virtually tuning free application. These results enable on-the-fly

\footnotetext{
${ }^{1}$ Navigation Function tuning refers to the process of determining the appropriate level of the parameter $k$ that will render the candidate Navigation Function (i) free of local minima and (ii) a Morse function.
}

addition, removal and re-arranging of obstacles without losing the Navigation Function properties. However these results are valid only for single robot navigation. An appropriate extension of those results is required to enable multi-robot navigation and this is what is provided in the current work.

Multi-robot NF based techniques that have appeared in the literature assume disk shaped robots even-though the multirobot NF theory does not exclude the possibility of robots diffeomorphic to spheres. One of the major drawbacks in handling obstacles under the framework of multi-robot navigation functions (MRNFs), is the absence of an appropriate transformation of arbitrarily shaped worlds to the spherical world where MRNFs were constructed. This paper is the first to the author's knowledge that treats multi-robot navigation in environments with arbitrarilly shaped obstacles under the Navigation Functions framework. Global knowledge regarding the location and geometry of each workspace entity is assumed to be available to every robot in this paper. In this paper robots are assumed to only have 2 degrees of freedom, hence only translational motion of robots is considered, whereas rotational motion is beyond the scope of this paper. The major contributions of the methodology proposed in this paper over the existing Multi-Robot Navigation Functions literature (MRNF) are as follows:

1) Correct by construction: Absence of local minima is inherited from the underlying harmonic function without need for tuning, whereas a tuning controller is implemented to establish the non-degeneracy of critical points.

2) On the fly addition and removal of workspace entities without need for tuning.

3) Handling of static obstacles. MRNF literature has demonstrated handling of controlled, sphere-shaped workspace entities. Static, arbitrarily shaped and arbitrarilly spaced obstacles have not been formally treated under MRNFs. These cases may yield grown obstacles that are non-spherical. Moreover grown obstacles may modify the number of disjoint workspace entities which would either require conservative tuning or re-tuning of MRNFs.

4) Handling of arbitrarilly shaped workspace entities through an appropriate transformation. MRNF literature has only demonstrated handling of sphere-shaped ob- 
jects, even though the MRNF theory does not explicitly exclude arbitrarily shaped objects.

The rest of the paper is organized as follows: Section II introduces preliminary notions, definitions and assumptions. Section III motivates and introduces the concept of adsorption mapping and proposes a construction for a candidate Multi-Agent Navigation Transformation. Section IV] presents the construction of a Harmonic Function based navigation Function, the controller design and system stability analysis. Section $\mathrm{V}$ presents simulation results whereas section VI concludes the paper.

\section{PRELIMINARIES}

In this section we introduce the necessary terminology and definitions for the development of the methodology.

If $K$ is a set, then $\stackrel{\circ}{K}$ is the interior, $\partial K$ the boundary and $(K)^{c}$ the complement of $K$. Also if set $K$ is finite, then $K^{i}$ denotes its $i$ 'th element whereas $|K|$ denotes its cardinality. Let $\bar{D}_{\rho}$ denote the closed disk with radius $\rho$ that is centered at the origin. We denote with $\nabla_{x_{i}} \phi_{i}$ the gradient of $\phi_{i}$ along the $x$ and $y$ components of $x_{i}$.

Definition 1: Let $P_{i} \in \mathbb{R}^{2}, i \in\{1, \ldots M\}$ be $M$ discrete elements of $\mathbb{R}^{2}$. Then a 2-D point world is defined as a manifold

$$
\mathcal{P} \subseteq \mathbb{R}^{2} \backslash \bigcup_{i=1}^{M} P_{i}
$$

Definition 2: A 2-D point world with spherical boundary is a manifold

$$
\tilde{\mathcal{P}} \subseteq \mathcal{P} \backslash\left(D_{\rho}\right)^{c}
$$

where $\bigcup_{i=1}^{M} P_{i} \in D_{\rho}$.

Definition 3: The workspace $\mathcal{W} \subset \mathbb{R}^{2}$ is a manifold such that $\mathcal{W}$ is diffeomorphic to $\tilde{\mathcal{P}}$ or $\mathcal{P}$.

Assume that our workspace is populated with entities comprising $n_{R}$ robots and $n_{s}$ obstacles. Let $\mathcal{R}, \mathcal{S}$ and $\mathcal{M}$ denote the sets of robots and obstacles respectively. Workspace entities are grouped in the set

$$
\mathcal{E} \triangleq \mathcal{R} \cup \mathcal{S} \cup \mathcal{M}
$$

The robots are assumed to be holonomic systems with kinematics as follows:

$$
\dot{q}_{i}=u_{i}, \quad i=1 \ldots n_{R}
$$

where $q_{i} \in \mathbb{R}^{2}$ is the reference point of robot $i$ and $u_{i}=$ $\left[\begin{array}{l}u_{x, i} \\ u_{y, i}\end{array}\right]$ it's velocity input. The destination configuration is denoted as $q_{d}^{i}$. The decentralized approach adopted in this paper is robot-centric, i.e. every robot has its own point of view of the world. Now assume that we treat robot $\eta$. The volume of robot $\eta$ is subtracted from robot $\eta$ and added to all the rest workspace entities (other robots, obstacles, external boundary). In that way robot $\eta$ treats the rest of the workspace entities as grown obstacles by taking the Minkowski sum $\oplus$ (see e.g. [11]) so that robot $\eta$ can be treated as a point.
Definition 4: Robot destination configurations physically blocking the path to the destination configuration of another robot for a given workspace, are called blocking arrangements.

Let $x(t)$ and $f(x, t)$ be $n$-dimensional vector valued functions. We need the following definitions from the Carathéodory differential equations literature:

Definition 5 (Carathéodory conditions [1] 57): In the domain $D$ of the $(x, t)$ space, function $f(x, t)$ has the following properties:

1) Continuity: For almost all $t, f(x, t)$ is continuous in $x$.

2) Measurability: For each $x, f(x, t)$ is measurable in $t$.

3) Boundedness: $\mid f(x, t \mid \leq m(t)$, where function $m(t)$ is summable (on each finite interval if $t$ is not bounded in the domain D).

Definition 6 (Carathéodory equation [5]): The equation

$$
\dot{x}=f(x, t)
$$

where $x$ is a scalar or a vector and the function $f$ satisfies the conditions of Definition 5] is called the Carathéodory equation.

Definition 7 (Carathéodory solution concept [5]): A function $x(t)$ defined on an open or closed interval $l$ is called a solution of the Carathéodory equation if it is absolutely continuous on each closed interval $[\alpha, \beta] \subset l$ and satisfies almost everywhere equation (2) or, which under the conditions in Definition 5 is the same thing, if it satisfies the integral equation:

$$
x(t)=x\left(t_{0}\right)+\int_{t_{0}}^{t} f(x(s), s) d s
$$

for some $t_{0} \in l$

In order to formulate the problem, we need the following assumptions:

Assumption 1: .

a. Obstacles and robot destination configurations do not form blocking arrangements.

b. At destination configurations workspace entities have a non-zero distance between each other.

Assumption 1 1 a is required to ensure that the destination configuration of each robot is reachable.

Assumption 1 b is required since workspace entities are not point-sized and they keep on occupying their workspace portion even when they arrive at their destination.

An additional assumption is required:

Assumption 2: Workspace entities are formed as finite unions of convex objects that admit smooth (at least $C^{2}$ ) distance metrics from their boundaries.

Assumption 2 does not pose practical limitations since any possible shape can be approximated arbitrarilly well (but not perfectly) by the union of finite convex objects.

Problem Statement: Given workspace $\mathcal{W}$ populated with arbitrarily shaped static obstacles and a set of arbitrarily shaped robots with kinematics as defined in (1). Assuming that each robot has perfect knowledge of its state and the state of the environment only, and that Assumptions 1 and 2 hold. 
Determine a decentralized feedback based control law to be executed on each and every robot to safely steer the robot away from collisions with other workspace entities and to it's destination configuration.

\section{Multi-Agent Navigation Transformation}

\section{A. Adsorption Mapping}

As workspace entities evolve, the purpose of the multi-agent Navigation Transformation (maNT) is to map their boundaries to appropriate locations in $\tilde{\mathcal{P}}$ or $\mathcal{P}$ (depending whether the workspace is bounded) so that a Harmonic Function based NF can be constructed. However one of the major issues in the multi-robot plus static obstacles (MRSO) problem that probably hindered previous developments of a "MRSO" sphereworld transformation is that, contrary to the static-environment single-robot case, its not only the position of the images under the transformation of the workspace entities that need to be dynamically modified to correspond to the current location of workspace entities. It is also the topology i.e. the Euler characteristic of the workspace that is changing. Although shifting the image position is straightforward, dynamically modifying the transformation to correspond to the dynamically changing topology is non-trivial. To see this consider two workspace entities approaching each other and eventually intersecting (e.g. in the case of grown obstacles from the $i$ 'th agent's point of view). Initially the two entities are two distinct topological entities, however when they intersect the two distinct topological entities merge into one. Intuitively to be able to exploit the concept of navigation transformation in the case of multiple robots, we need a transformation with the capability to adsorb ${ }^{2}$ the volume of the two intersecting entities and collapse them to a single point in $\mathcal{P}$ (or $\tilde{\mathcal{P}}$ ). This behavior, termed the adsorption mapping, is captured by the maNT.

Assume now that we are treating the problem from the $\eta$ 'th robot's point of view. Whenever not necessary, the robot index will be dropped for notational brevity. Let

$$
\bar{\eta}=\{\varepsilon \oplus \eta \mid \varepsilon \in \mathcal{E} \backslash \eta\}
$$

denote the set of grown workspace entities resulting from the Minkowski sum with robot $\eta$ and let $\mathcal{W}_{\eta}$ denote the grown workspace from agent $\eta$ 's point of view (see Figure 11).

Assuming for analysis purposes an indexing of the elements of $\bar{\eta}$, then its $i$ 'th element is denoted as $\bar{\eta}_{i}$, where $i \in \mathcal{I}_{\bar{\eta}}$ and $\mathcal{I}_{\bar{\eta}}$ is the index set of $\bar{\eta}$. Let $d_{i j}$ denote a distance metric between $\partial \bar{\eta}_{i}$ and $\partial \bar{\eta}_{j}$. Let $P_{\mathcal{W}}^{i} \in \mathcal{W}$ be the reference point of $\bar{\eta}_{i}$ and let $q$ to be the reference point of robot $\eta$.

Define the edge set

$$
E_{\bar{\eta}} \triangleq\left\{(i, j) \mid i, j \in \mathcal{I}_{\bar{\eta}} \wedge d_{i j}=0\right\}
$$

and the vertex set

$$
V_{\bar{\eta}} \triangleq\left\{i \mid \exists j \in \mathcal{I}_{\bar{\eta}}:(i, j) \in E_{\bar{\eta}} \vee(j, i) \in E_{\bar{\eta}}\right\} .
$$

\footnotetext{
2 adsorption: the capability of a substance to attract to its surface molecules of substances that are in contact
}

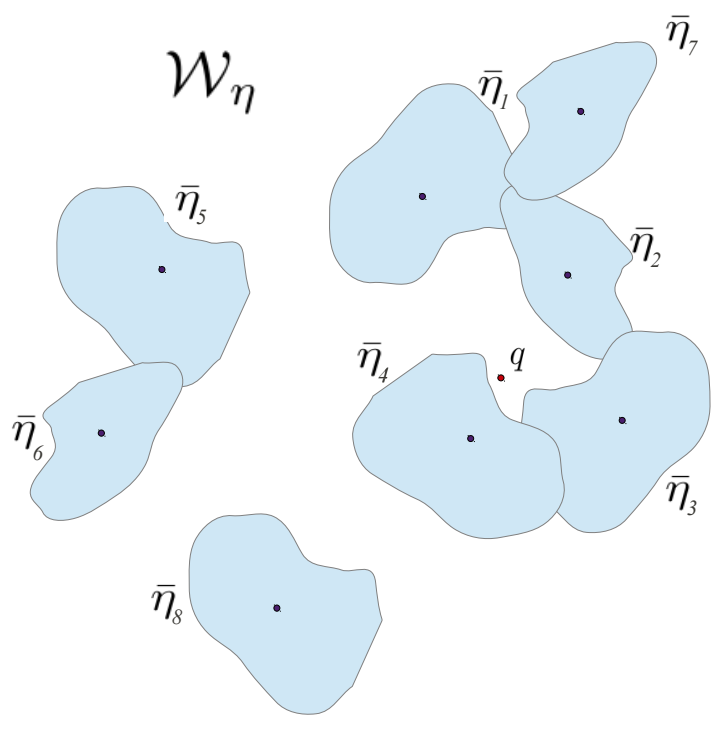

Fig. 1. A grown workspace $\mathcal{W}_{\eta}$ with eight workspace entities $\left(\bar{\eta}_{1}, \ldots \bar{\eta}_{8}\right)$ plus the robot $\eta$ with reference point $q$.

Then the graph of intersecting grown entities is defined as:

$$
\mathcal{G}_{\bar{\eta}} \triangleq\left\{V_{\bar{\eta}}, E_{\bar{\eta}}\right\}
$$

Since there might be chains of intersecting grown entities we need to identify the connected components in $\mathcal{G}_{\bar{\eta}}$ (see also Fig. 2). Assume the set $\mathcal{C}_{\bar{\eta}} \in 2^{\mathcal{G}_{\bar{\eta}}}$ containing all the connected components of $\mathcal{G}_{\bar{\eta}}$. Construct the set $\mathcal{T}_{\bar{\eta}}$ such that $\mathcal{T}_{\bar{\eta}}^{i}$ is a spanning tre ${ }^{3}$ of $\mathcal{C}_{\bar{\eta}}^{i}$. Construct the set $\mathcal{M}_{\bar{\eta}}$ such that $\mathcal{M}_{\bar{\eta}}^{i}$ is the first element of the graph center ${ }^{4}$ of $\mathcal{T}_{\bar{\eta}}^{i}$. Construct the set $\mathcal{D}_{\bar{\eta}}$ such that $\mathcal{D}_{\bar{\eta}}^{i}$ is defined by rooting $\mathcal{T}_{\bar{\eta}}^{i}$ at vertex $\mathcal{M}_{\bar{\eta}}^{i}$, assigning it as a sink and enforcing the implied orientation. Let the operator

$$
P:\left\{1, \ldots\left|\mathcal{D}_{\bar{\eta}}\right|\right\} \times\left\{\mathcal{D}_{\bar{\eta}}^{i} \cap V_{\bar{\eta}}\right\} \rightarrow\left\{\mathcal{D}_{\bar{\eta}}^{i} \cap V_{\bar{\eta}}\right\}
$$

return the directed path $\Pi$ from vertex $v$ of $\mathcal{D}_{\bar{\eta}}^{i}$ to the root $\mathcal{M}_{\bar{\eta}}^{i}$, such that $\Pi^{1}=v, \Pi^{|\Pi|}=\mathcal{M}_{\bar{\eta}}^{i}$ and for any $i \in\{1, \ldots|\Pi|-1\}$ we have that $\left\{\Pi^{i}, \Pi^{i+1}\right\} \in \mathcal{D}_{\bar{\eta}}^{i}$.

With Assumption 1, let $b_{i}(x), i \in \mathcal{I}_{\bar{\eta}}$, be a smooth (at least $C^{2}$ ) monotonically increasing function with respect to the distance of point $x$ from the boundary of $\eta_{i}$ and $b_{i}\left(\partial \eta_{i}\right)=0$. Also define the distance to the goal function of agent $\eta$ as

$$
\gamma_{d}^{\eta}(q)=\left\|q-q_{d}^{\eta}\right\|^{2}
$$

Define the smooth switch function:

$$
\eta(x, y) \triangleq \frac{s(x)}{s(x)+s(y-x)}
$$

\footnotetext{
${ }^{3}$ Note that although cyclic graphs have multiple spanning trees, it is assumed that a deterministic algorithms will return one specific $\mathcal{C}_{\bar{\eta}}^{t, i}$ for the same $\mathcal{C}_{\bar{\eta}}^{i}$ input

${ }^{4}$ It is a well known result by C.Jordan[7] that the center of a tree graph consists either of one vertex or two adjacent vertices
} 


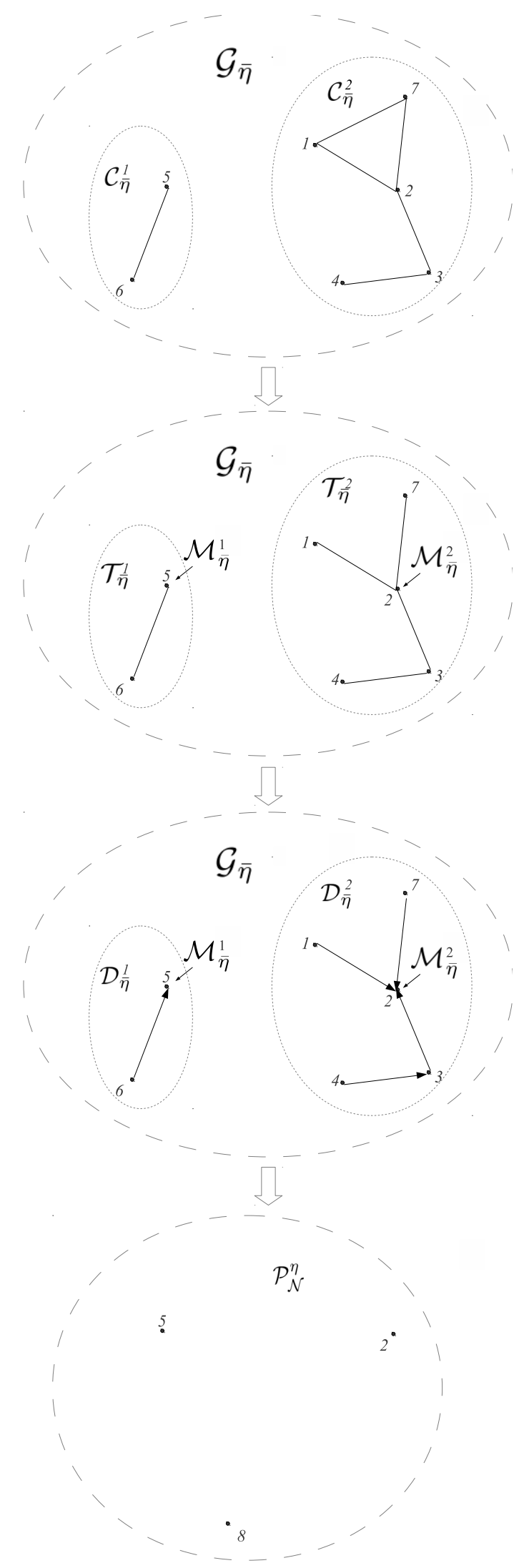

Fig. 2. For the workspace $\mathcal{W}_{\eta}$ depicted in Fig. 1 we have the intersecting grown entities graph $\mathcal{G}_{\bar{\eta}}$ and subsequently the sets $\mathcal{C}_{\bar{\eta}}, \mathcal{T}_{\bar{\eta}}, \mathcal{M}_{\bar{\eta}}, \mathcal{D}_{\bar{\eta}}$ are defined according to the methodology presented in Section III-A Using the maNT, $\mathcal{W}_{\eta}$ is eventually mapped to $\mathcal{P}_{\mathcal{N}}^{\eta}\left(\right.$ or $\tilde{\mathcal{P}}_{\mathcal{N}}^{\eta}$ ) as described in Section III-B where

$$
s(t) \triangleq \begin{cases}e^{-1 / t} & t>0 \\ 0 & t \leq 0\end{cases}
$$

Define the function:

$$
\eta_{a}\left(x, y_{1}, \ldots y_{n}\right) \triangleq \prod_{i=1}^{n} \frac{s\left(\frac{y_{i}-x}{\epsilon}+\frac{1}{2}\right)}{s\left(\frac{y_{i}-x}{\epsilon}+\frac{1}{2}\right)+s\left(\frac{x-y_{i}}{\epsilon}+\frac{1}{2}\right)}
$$

where $\epsilon>0$ is a small positive constant. Function $\eta_{a}$ becomes 1 if $x$ is smaller than all $y_{i}-\epsilon / 2$ and zero if $x$ is larger than any $y_{i}+\epsilon / 2$. Constant $\epsilon$ introduces a technically necessary transition region in order to make (4) smooth. This function induces locally a Voronoi-like workspace partitioning enabling the adsorption mapping to be initiated by the obstacle that is closest to the robot.

Define $\delta$ to be the influence region of each workspace entity. The influence region was initially introduced in [13], to capture various features of the robots, like limited sensing and regions necessary for abstracting kinematic and dynamic constraints and was a feature of the Navigation Transformation. In this paper global knowledge is assumed. Practically only knowledge of location and geometry of the connected components (entities) to the proximal entity is required, since these are necessary to perform the adsorption mapping. These components, though, could span across the entire workspace. This is equivalent to the case in single robot navigation where the robot approaches a big obstacle that spans across the workspace, so "local" knowledge about such an obstacle (i.e. location plus geometry) implies knowledge about the state of a significant portion of the workspace.

Define the function

$$
\sigma_{1}(x) \triangleq \frac{x}{\delta}(1-\eta(x, \delta))+\eta(x, \delta)
$$

Then define the adsorption transformation as:

$$
v_{i}(q, b) \triangleq\left(1-\sigma_{1}(b)\right)\left(P_{\mathcal{W}}^{i}-q\right)
$$

The path adsorption for vertex $v=\Pi^{1}$ along path $\Pi$ is defined as follows:

$$
U_{\Pi}(b, q) \triangleq v_{\Pi|\Pi|}\left(v_{\Pi^{|\Pi|-1}}\left(\ldots\left(v_{\Pi^{1}}(q, b), b\right), \ldots, b\right), b\right)
$$

Eventually the adsorption mapping for the $i$ 'th component of $\mathcal{D}_{\bar{\eta}}$ is defined as the linear combination of path adsorptions along the directed paths initiating from every vertex $v \in \mathcal{D}_{\bar{\eta}}^{i}$ :

$$
C_{\mathcal{D}_{\bar{\eta}}}^{i}(q)=\sum_{\substack{\Pi=P(i, v) \\ v \in \mathcal{D}_{\bar{\eta}}^{i}}} \eta_{a}\left(b_{v}, \bar{b}_{v}^{\gamma}\right) U_{\Pi}\left(b_{v}, q\right)
$$

where

$$
\bar{b}_{v}^{\gamma}=\left\{b_{1}, \ldots b_{v-1}, b_{v+1}, \ldots b_{\left|\mathcal{I}_{\eta}\right|}, \gamma_{d}^{\eta}\right\} .
$$

The reason of including $\gamma_{d}^{\eta}$ in $\bar{b}_{v}^{\gamma}$ is to enable the adsorption mapping to diminish as the robot gets closer to it's destination. 


\section{B. Multi-Agent Navigation Transformation (maNT)}

Before proceeding with the present of the maNT we will need a few more definitions: Let

$$
\mathcal{N}_{\eta} \triangleq\left\{\mathcal{I}_{\bar{\eta}}-V_{\bar{\eta}}\right\} \cup \mathcal{M}_{\bar{\eta}} .
$$

Index $\eta$ will be dropped in the sequel for $\mathcal{N}_{\eta}$ for notational brevity. Let us define the following 2-D point worlds:

$$
\mathcal{P}_{\mathcal{N}}^{\eta} \subseteq \mathbb{R}^{2} \backslash \bigcup_{v \in \mathcal{N}} P_{\mathcal{W}}^{v}
$$

and

$$
\tilde{\mathcal{P}}_{\mathcal{N}}^{\eta} \subseteq \mathcal{P}_{\mathcal{N}}^{\eta} \backslash\left(D_{\rho}\right)^{c} .
$$

$\mathcal{P}_{\mathcal{N}}^{\eta}$ will be referred to as the harmonic domain whereas $\tilde{\mathcal{P}}_{\mathcal{N}}^{\eta}$ is the 2-D point world where a bounded workspace is mapped through the maNT, both as seen from agent $\eta$ 's point of view. The Multi-Agent Navigation Transformation is defined as follows:

Definition 8: The Multi-Agent Navigation Transformation is a diffeomorphism $T_{\eta}: \mathcal{L}_{\eta} \rightarrow \mathcal{P}_{\mathcal{N}}^{\eta}\left(\right.$ or $\left.T_{\eta}: \stackrel{\mathcal{W}}{\eta}_{\eta} \rightarrow \tilde{\mathcal{P}}_{\mathcal{N}}^{\eta}\right)$.

According to the above definition, the Multi-Agent Navigation Transformation is a multi-transformation that maps the interior of the grown workspace $\mathcal{W}_{\eta}$ to multiple topologies $\mathcal{P}_{\mathcal{N}}^{\eta}\left(\right.$ or $\tilde{\mathcal{P}}_{\mathcal{N}}^{\eta}$ ) dictated by the set $\mathcal{N}$ as defined in 5 .

With Assumption 2, the following transformation is proposed as a candidate Multi-Agent Navigation Transformation:

$$
\begin{aligned}
T_{\eta}(q)= & \operatorname{id}(q)+\sum_{i \in\left\{1 \ldots\left|\mathcal{D}_{\bar{\eta}}\right|\right\}} C_{\mathcal{D}_{\bar{\eta}}}^{i}(q) \ldots \\
& +\sum_{\mu \in\left\{\mathcal{I}_{\bar{\eta}}-V_{\bar{\eta}}\right\}} \eta_{a}\left(b_{\mu}, \bar{b}_{\mu}^{\gamma}\right) v_{\mu}\left(q, b_{\mu}\right)
\end{aligned}
$$

The proposed candidate maNT is of the form $T_{\eta}: \stackrel{\mathcal{W}}{\eta}_{\eta} \rightarrow$ $\mathcal{P}_{\mathcal{N}}^{\eta}$. An appropriate maNT of the type $T_{\eta}: \stackrel{\circ}{\mathcal{W}}_{\eta} \rightarrow \tilde{\mathcal{P}}_{\mathcal{N}}^{\eta}$ requires an additional step for contracting grown workspace entities that are in contact with the boundary, to the boundary - and this step is not tackled in the current paper. Theoretical analysis of the properties of the proposed transformation (6) is beyond the scope of the current paper and is the subject of a separate paper.

Figure 3 demonstrates conceptually how the position $q$ of agent $\eta$ is mapped to a location in $\mathcal{P}_{\mathcal{N}}^{\eta}$ through transformation (6).

\section{HFNF BASEd CONTROLLER DESIGN}

\section{A. Construction of the Harmonic Function based Navigation Function}

The Harmonic Function based Navigation Function (HFNF) that is implemented is the one proposed by Loizou [12]. For the completeness of presentation, some basic facts are repeated here:

The HFNF $\Theta_{\eta}^{\mathcal{N}}$ will be different for each robot $\eta$ and is defined based on the underlying point world. For maNT of the type $T_{\eta}: \stackrel{\circ}{\mathcal{V}}_{\eta} \rightarrow \mathcal{P}_{\mathcal{N}}^{\eta}$ it is a composition of the following:

$$
\Theta_{\eta}^{\mathcal{N}}(\cdot)=\left[\sigma_{d} \circ \sigma \circ \phi_{\eta}^{\mathcal{N}} \circ T_{\eta}\right](\cdot) .
$$

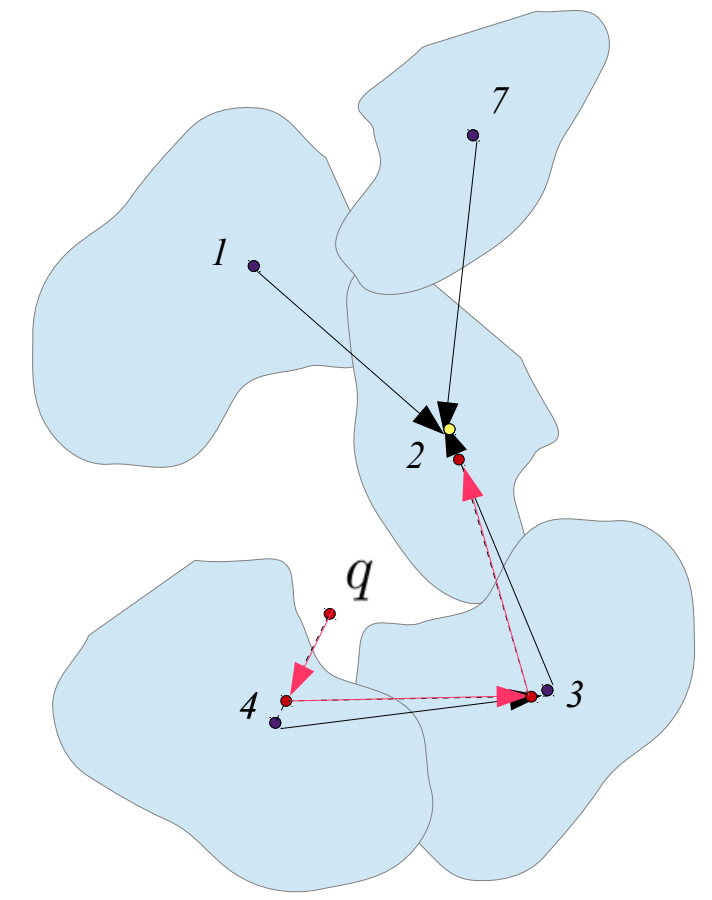

Fig. 3. Mapping $q$ to $\mathcal{P}_{\mathcal{N}}^{\eta}$ through the maNT proposed in 6. Depicted is the adsorption mapping of agent $\eta$ located at $q$ along the reference points of entities $\{4,3\}$ to the root-entity $\{2\}$

For maNT of the type $T_{\eta}: \stackrel{\mathcal{W}}{\eta}_{\eta} \rightarrow \tilde{\mathcal{P}}_{\mathcal{N}}^{\eta}$ it is a composition of the following:

$$
\Theta_{\eta}^{\mathcal{N}}(\cdot)=\left[\sigma_{d} \circ \sigma \circ \phi_{\eta}^{\mathcal{N}} \circ c^{-1} \circ T_{\eta}\right](\cdot)
$$

where $T$ is a multi-agent Navigation Transformation. The transformation $c^{-1}: \tilde{\mathcal{P}}_{\mathcal{N}}^{\eta} \rightarrow \mathcal{P}_{\mathcal{N}}^{\eta}$ that takes the form:

$$
c^{-1}(x)=\frac{x}{\rho-\|x\|}
$$

maps the 2-D point world $\tilde{\mathcal{P}}_{\mathcal{N}}^{\eta}$ to the harmonic domain $\mathcal{P}_{\mathcal{N}}^{\eta}$. Note that in this case, the destination configuration should be mapped to the corresponding location in the harmonic domain, i.e. $h_{d}=c^{-1}\left(q_{d}\right)$. Function $\phi_{\eta}^{\mathcal{N}}$ is the underlying harmonic function defined as

$$
\phi_{\eta}^{\mathcal{N}}(h, t)=\phi_{d}(h)+\sum_{i \in \mathcal{N}} \phi_{i}(h)
$$

where $\mathcal{N}$ as defined in eq. (5) is the set of adsorption points mapped in $\mathcal{P}_{\mathcal{N}}^{\eta}$. Note that since the set $\mathcal{N}$ changes over time, $\phi_{\eta}^{\mathcal{N}}$ exhibits discontinuities in $t$, but is smooth in $h$.

Function

$$
\phi_{d}(h)=(1+M) \ln \left(\left\|h-h_{d}\right\|\right)
$$

is the harmonic destination potential. According to the theoretical analysis presented in [12] the requirements on $M$ are that $M \geq|\mathcal{N}|$. Hence taking a conservative approach we can use $M=|\mathcal{E}|$. Functions:

$$
\phi_{i}(h)=-\ln \left(\left\|h-h_{i}\right\|\right)
$$


are the harmonic potential contributions from the $i$ 'th contracted workspace entity. In the case of a bounded workspace we have $h_{i}=c^{-1}\left(P_{\mathcal{W}}^{i}\right)$.

Function

$$
\sigma(x) \triangleq \frac{e^{x}}{1+e^{x}}
$$

maps the extended real number line to the interval $[0,1]$, whereas

$$
\sigma_{d}(x) \triangleq x^{2 / k}
$$

is a distortion function.

\section{B. Controllers}

The tuning controller proposed in [12] is implemented to establish the non-degeneracy of the critical points, by applying the parameter update law to each agent:

$$
\dot{k}_{\eta}=K_{t} \cdot H_{\varepsilon}\left(c_{0, \eta}\left(x_{\eta}\right)\right) \cdot H_{\varepsilon}\left(\left|\theta_{\eta}\left(x_{\eta}\right)\right|+\left|\eta_{0, \eta}\left(x_{\eta}\right)\right|\right)
$$

with $k_{\eta}(0)=M+1, \varepsilon \ll 1$ and $K_{t}$ a positive tuning parameter. More details are provided in [12].

Let $\mathcal{I}_{\tau} \sim \mathcal{U}\left(t_{z}, n t_{z}\right)$ be a uniformly distributed random variable on the interval $\left[t_{z}, n t_{z}\right]$, where $t_{z}>0$ and $n \geq 2$.

The control law that is applied to robot $\eta$ is given by:

$$
u_{\eta}\left(q_{\eta}, t\right)= \begin{cases}-K \nabla_{q_{\eta}} \Theta_{\mathcal{N}}^{\eta}\left(q_{\eta}, t\right), & \delta \geq t_{z} \\ \mathbf{0}, & \delta<t_{z}\end{cases}
$$

where $K$ a positive gain, $\tau_{\mathcal{N}}=\tau_{\mathcal{N}}(t)$ is the ordered set of time instants up to time $t$, when $\mathcal{N}$ was modified or $t:=$ $\tau_{\mathcal{N}}+\mathcal{I}_{\tau}$ while $\delta<t_{z}$, whereas

$$
\delta= \begin{cases}\delta(t) \triangleq \tau_{\mathcal{N}}^{\left|\tau_{\mathcal{N}}\right|}-\tau_{\mathcal{N}}^{\left|\tau_{\mathcal{N}}\right|-1}, & \left|\tau_{\mathcal{N}}\right| \geq 2 \\ 2 t_{z}, & \left|\tau_{\mathcal{N}}\right|<2\end{cases}
$$

Essentially control law (10) is the negated gradient flows of $\Theta_{\mathcal{N}}^{\eta}\left(q_{\eta}, t\right)$, augmented with a random dwell time in the case a Zeno-type behavior is detected.

\section{Stability Analysis}

We will start by stating some preliminary results

Lemma 1: System (1) with control law (10) forms a Carathéodory equation

Proof: Due to space constraints only a sketch of proof is provided.

According to Definition 6 we need to show that the system with the control law satisfies the conditions of Definition 5.

Continuity: Since $\Theta_{\mathcal{N}}^{\eta}\left(q_{\eta}, t\right)$ is a composition of smooth functions, its negated gradient vector field will also be smooth and hence continuous in $q_{\eta}$. Now for the almost everywhere in $t$ continuity, since no Zeno-type behavior is allowed in the system when the number of entities is modified due to the random dwell time introduced by controller 10 no infinite switching is possible and the switching instances are a set of measure zero.

Measurability: Since for each $x, f(\cdot, t)$ is an almost everywhere continuous function defined on measurable sets, it will also be measurable since, since continuous functions defined on measurable sets are measurable functions and sets of measure zero do not affect the measurability of a function [2].

Boundedness: Since the time domain is unbounded we only need to show that on each finite interval $\Omega$ of the non-negative real line, the integral

$$
\int_{\Omega} \nabla_{q_{\eta}} \Theta_{\mathcal{N}}^{\eta}\left(q_{\eta}, t\right) d t<+\infty
$$

exists and is finite. Observing that $\nabla_{q_{\eta}} \Theta_{\mathcal{N}}^{\eta}\left(q_{\eta}, t\right)$ is bounded (observe this by expanding $\sigma_{d} \circ \sigma \circ \phi_{\eta}^{\mathcal{N}}$ and taking the gradient) implies that the the integral is bounded on each finite interval as required.

The following is the main result of the paper:

Proposition 1: System (1) with Assumption 1, under the parameter update laws (9) and under the control laws (10), is globally asymptotically stable, almost everywhere.

Proof: Due to space constraints only a sketch of proof is provided.

Step 1: Positive invariance of $\mathcal{W}$ : Due to the uniform maximality property of navigation functions, the gradient flow is perpendicular to the workspace boundary and this implies that robots are repelled by the workspace boundary. Hence $\mathcal{W}$ is a positively invariant set.

Step 2: Types of $\omega_{\eta}$ - limit sets: Since $\mathcal{W}$ is positively invariant and each robot has a unique global attractor and a finite number of non-degenerate saddle points, this implies that $\omega_{\eta} \subset \stackrel{\circ}{\mathcal{W}}$. Now assume that for the trajectory $\tau_{\eta}$ of robot $\eta$, there are $t_{a}, T>0$ such that $\tau_{\eta}\left(t_{a}\right)=\tau_{\eta}\left(t_{a}+T\right)$ and the trajectory $\tau_{\eta}(t), t \in\left[t_{a}, T\right]$ is a Jordan curve. Since according to Lemma 1, system (1) with control law (10) forms a Carathéodory equation, it will satisfy the integral equation in (3). Hence we have that

$$
\int_{t_{a}}^{t_{a}+T} \dot{\Theta}_{\mathcal{N}^{-}}^{\eta} \mathrm{d} t=0
$$

So

$$
\int_{t_{a}}^{t_{a}+T} \frac{1}{K}\left\|\dot{x}_{i}\right\|^{2} \mathrm{~d} t=\sum_{j \neq i} \int_{t_{a}}^{t_{a}+T} \nabla_{x_{j}} \Theta_{\mathcal{N}}^{i} \dot{x}_{j} \mathrm{~d} t
$$

This implies that at least one of $\nabla_{x_{j}} \Theta_{\mathcal{N}^{i}}^{i} \dot{x}_{j}$ is persistently positive over some time interval and $x_{j}$ is in the close proximity of $x_{i}$ during that interval. This implies that $\dot{x}_{j}$ and $\dot{x}_{i}$ will have directions in the same quadrant. Now observe that $\varphi_{i}$ increases whenever $x_{j}$ moves towards $x_{i}$ and vice versa, hence $\nabla_{x_{j}} \varphi_{i}$ and $\nabla_{x_{i}} \varphi_{j}$ will be pointing in opposite directions, away from $i, j$. This in turn implies an ordering, more specifically that $i$ leads and $j$ follows. From the $j$ 'th agent's point of view based on the above reasoning, $\nabla_{x_{i}} \varphi_{j} \dot{x}_{i}$ will be negative. However for the equivalent of equation (11) for agent $j$ to hold, using the same reasoning, agent $k$ has to follow agent $j$ and so on. However since we have a finite number of agents, the last agent in the reasoning will not have an agent to render the equivalent of eq. (11) true. Hence this agent will not be able to transverse a Jordan curve and will be asymptotically stable at its destination. Backtracking on our reasoning, no agent will 
be able to maintain a trajectory on a Jordan curve as an orbit, hence the $\omega$-limit set of each agent contains only the set of non-degenerate saddle points and the destination configuration. This in turns implies the global - almost everywhere (due to the existence of non-degenerate saddle points) - asymptotic stability of the destination configurations.

\section{Simulation Results}

To demonstrate the effectiveness of the proposed approach a simulation has been set up with 7 workspace entities, comprising 5 robots and 2 static obstacles as in Fig. 4 . Workspace entities were selected as unions of disks to approximate convex and non-convex shapes.

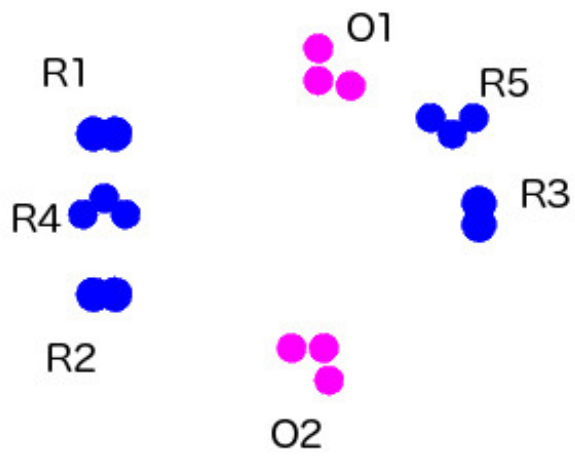

Fig. 4. A workspace with 5 robots $\left(R_{1}, \ldots R_{5}\right.$ in blue $)$ and two obstacles $\left(O_{1}, O_{2}\right.$ in magenta): Initial configuration.

Figure 5 depicts the destination configuration and figure 6 depicts the trajectories of the system.

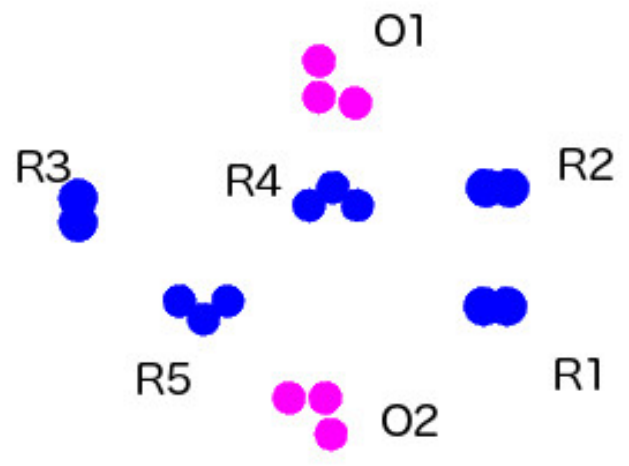

Fig. 5. Destination configuration

Figures 7 and 8 depict snapshots from intermediate configurations. As can be seen from the resulting system trajectories, the robots were safely navigated from their initial configuration to their destination avoiding collisions.

Remark It should be noted that the random dwell time branch of controller (10) was not activated during the simulation. As the random dwell time was utilized to facilitate

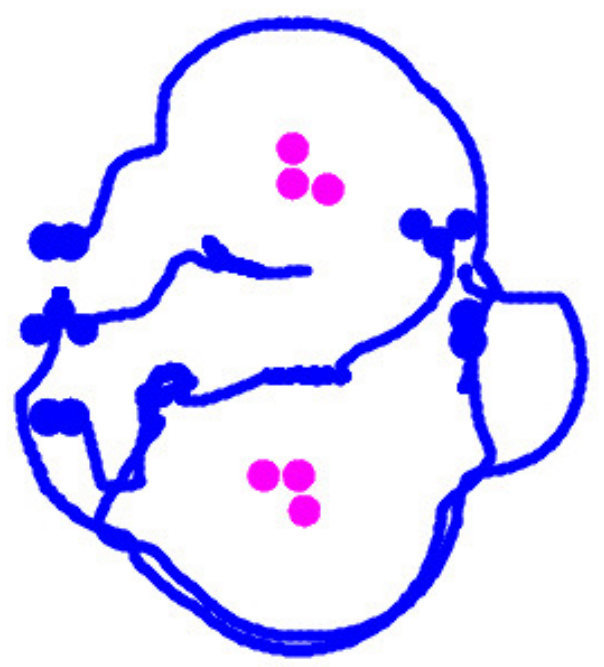

Fig. 6. System trajectories

the theoretical analysis, a more thorough investigation on the initial conditions that trigger its activation is part of ongoing work and beyond the scope of the current paper.

\section{CONClusions AND Further RESEARCH}

This paper presents the first to the author's knowledge solution to the problem of multi-robot navigation in environments with arbitrarily shaped obstacles under the Navigation Functions framework. The Navigation Transformation for multi-agent systems is defined and a candidate multi-agent Navigation Transformation is proposed. The proposed multiagent Navigation Transformation can handle the emerging topology of the workspace as the Euler characteristic of the workspace is changing due to the merging and splitting of the grown-obstacle image of dynamic workspace entities. Theoretical stability guarantees are provided and non-trivial simulation results verify the feasibility of the methodology.

Future research directions include extending the solution to account for dynamic obstacles, robot orientations, solving the problem in higher dimensions and studying the case of human-in-the-loop interactions. Extension of the proposed methodology to more realistic scenarios with local sensing and knowledge is currently being considered.

\section{ACKNOWLEDGMENTS}

This work was co-funded by the European Regional Development Fund and the Republic of Cyprus through the Research Promotion Foundation under research grant АЕІФОРІА/ГЕ $\Omega$ РГО/0311(BIE)/08.

\section{REFERENCES}

[1] D. C. Biles and P. A. Binding. On Carathéodory's conditions for the initial value problem. Proceedings of the American Mathematical Society, 125(5):pp. 13711376, 1997. URL http://www.jstor.org/stable/2162080. 


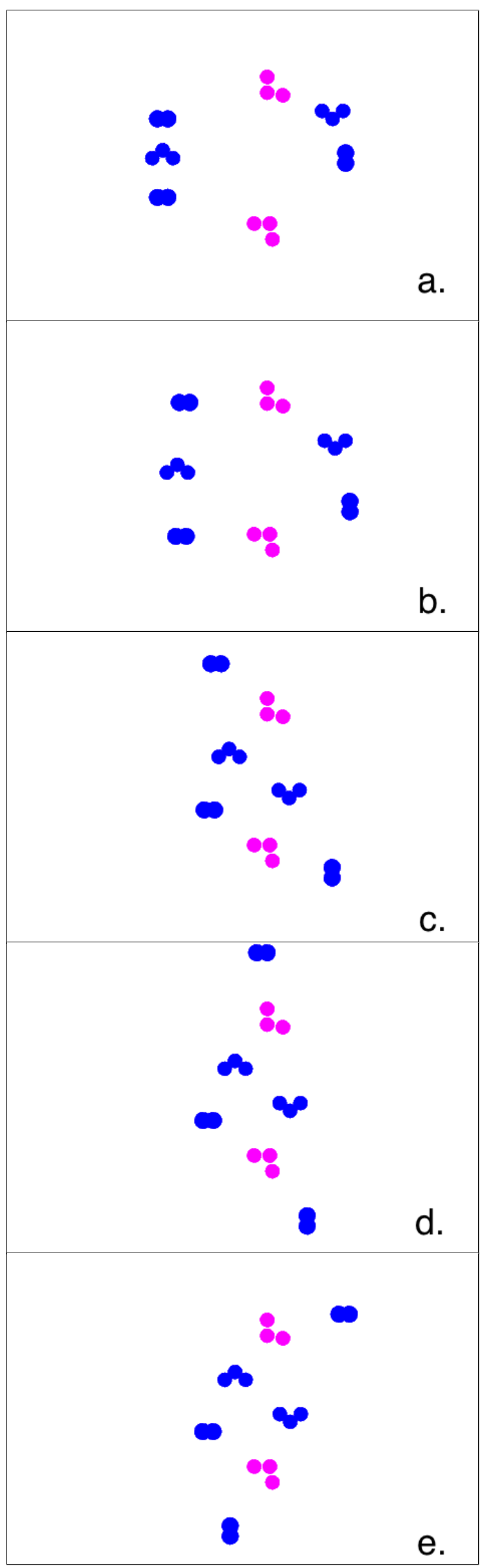

Fig. 7. Snapshots from intermediate configurations

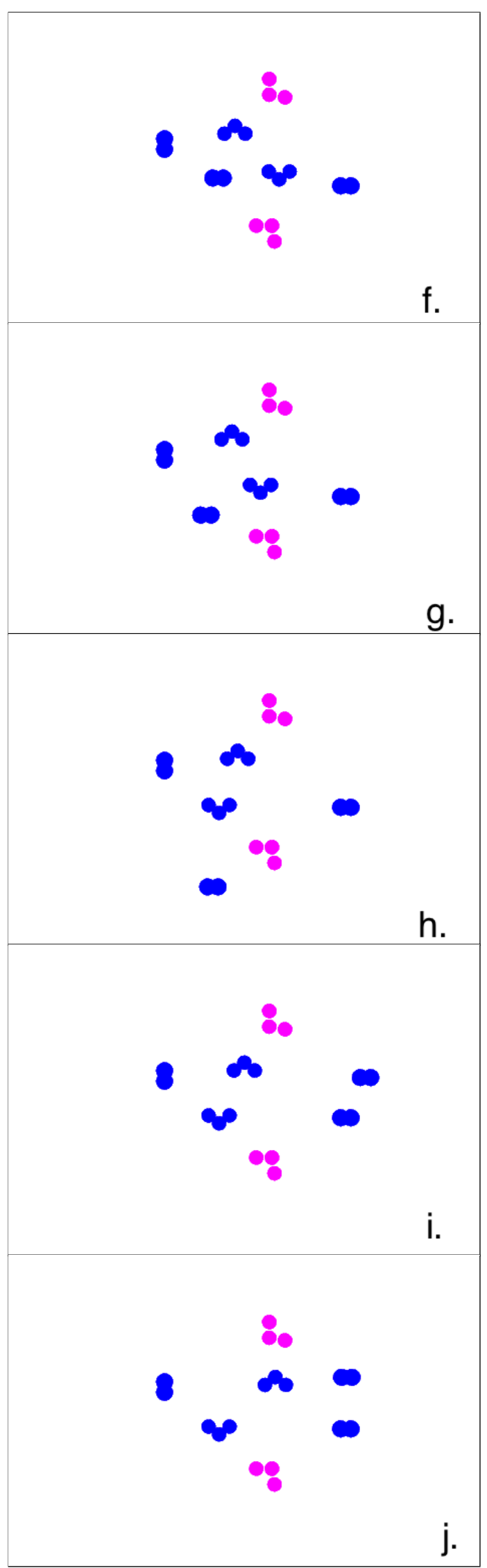

Fig. 8. Snapshots from intermediate configurations 
[2] Frank Burk. Lebesgue measure and integration : an introduction. Wiley Interscience, 1998.

[3] Dimos V. Dimarogonas. Sufficient conditions for decentralized potential functions based controllers using canonical vector fields. IEEE Trans. Automat. Contr, 57 (10):2621-2626, 2012. URL http://dx.doi.org/10.1109/ TAC.2012.2191319.

[4] Ioannis Filippidis, Dimos V. Dimarogonas, and Kostas J. Kyriakopoulos. Decentralized multi-agent control from local 1tl specifications. In 51 $1^{\text {st }}$ IEEE Conference on Decision and Control (CDC 2012), pages 6235 - 6240, Maui, Hawaii, USA, December 10-13 2012. doi: 10. 1109/CDC.2012.6426027.

[5] A. Filippov. Differential equations with discontinuous right-hand sides. Kluwer Academic Publishers, 1988.

[6] Meng Guo, Karl Henrik Johansson, and Dimos V. Dimarogonas. Motion and action planning under ltl specifications using navigation functions and action description language. In IROS, pages 240-245. IEEE, 2013. URL http://dx.doi.org/10.1109/IROS.2013.6696359

[7] C. Jordan. Sur les assemblages de lignes. J. Reine Angew. Math., 70:193-200, 1869.

[8] M. Kloetzer, X.C. Ding, and C. Belta. Multi-robot deployment from ltl specifications with reduced communication. IEEE Conference on Decision and Control, 2011.

[9] D. E. Koditschek and E. Rimon. Robot navigation functions on manifolds with boundary. Advances Appl. Math., 11:412-442, 1990.

[10] H. Kress-Gazit, D.C. Conner, H. Choset, A.A. Rizzi, and
G.J. Pappas. Courteous cars: Decentralized multiagent traffic coordination. Robotics Automation Magazine, IEEE, 15(1):30-38, 2008.

[11] J. C. Latombe. Robot Motion Planning. Kluwer Academic Publishers, 1991.

[12] S.G. Loizou. Closed form navigation functions based on harmonic potentials. 50th IEEE Conference on Decision and Control and European Control Conference, 2011.

[13] S.G. Loizou. The navigation transformation: Point worlds, time abstractions and towards tuning-free navigation. The Mediterranean Conference on Control and Automation, 2011.

[14] S.G. Loizou and K.J. Kyriakopoulos. Navigation of multiple kinematically constrained robots. IEEE Transactions on Robotics, 24(1):221-231, Feb. 2008.

[15] H. G. Tanner and A. Boddu. Multiagent navigation functions revisited. Robotics, IEEE Transactions on, 28 (6):1346-1359, 2012.

[16] H.G. Tanner and A.Kumar. Formation stabilization of multiple agents using decentralized navigation functions. Robotics: Science and Systems I, S. Thrun, G. Sukhatme, S. Schaal and O. Brock (eds), MIT Press, pages 49-56, 2005.

[17] L.L. Whitcomb and D.E. Koditschek. Automatic assembly planning and control via potential functions. Proceedings of the IEEE/RSJ International Workshop on Intelligent Robots and Systems, 1:17-23, 1991.

[18] J. Yu and S. M. LaValle. Multi-agent path planning and network flow. Workshop on the Algorithmic Foundations of Robotics, 2012. 